\title{
Histopathology and cytopathology of cervical cancer
}

\author{
David Jenkins \\ Glaxo Smith Kline Biologicals, Rixensart, Belgium \\ University of Nottingham, Nottingham, UK \\ E-mail: David.D.Jenkins@gskbio.com
}

\section{Introduction}

Histopathology and cytopathology form the scientific and clinical basis for current prevention and treatment of cervical cancer. Histopathology determines treatment of cancer and precancer through classifying into a diagnosis the patterns of microscopic organization of cells in tissue sections from biopsy or surgical specimens. Although morphological concepts of cervical cancer and precancer evolution are giving way to viral and molecular knowledge, histopathology also remains important as the most widely used clinical endpoints by which the performance of new techniques for cervical cancer prevention are currently evaluated. Cervical cytopathology studies exfoliated cells taken from the surface of the cervix and is the main method of cervical screening in successful cervical cancer prevention programmes.

These techniques have contributed hugely to reducing the burden of cervical cancer. Countries with successful implementation of cytological screening

have shown a reduction in incidence and mortality by $70 \%$ or more [1,2]. These figures are historical comparisons and may underestimate the impact as they do not take account of observed increased rates in young women for whom screening provides little protection, and the impact of increasing exposure to oncogenic HPV and other risk factors in some countries as a consequence of major behavioural changes. On this basis, in the UK, the national programme started in 1988 has been estimated to prevent 4,500-6,000 deaths per year, with a very marked decline in [3] rates in women aged
30-34 y. Despite this, because of the difficulties and costs of this approach, globally, there are almost half a million cases of cervical cancer each year.

Concurrently with the enormous success of cytological screening, there has been increasing knowledge of the challenges of the limited reproducibility of the diagnoses, of the complex relationships between cytological and histological diagnosis and the natural history of cervical precancer, particularly the driving role of infection with genital human papillomavirus (HPV).

This review focuses on the concepts and terminology used in classifying morphological changes of cervical precancer and HPV infection, how this links to natural history through information from cervical screening and more recent cohort studies of HPV infection. It addresses the issues around the performance characteristics (reproducibility and accuracy) of cytopathology and histopathology as diagnostic tests, and the implications for cervical screening practice. Finally it discusses the use of cytopathology and histopathology as reference standards and endpoints in clinical research and biomarker development.

\section{Historical background}

Histopathology has defined historically the concepts of development and progression of cervical precancer to cancer. The idea of carcinoma-in-situ as a cancer precursor dates back to the early cellular pathology of Rudolf Virchow during the nineteenth century. The concept of cervical precancer has been refined 
over more than a century to include less severe, dysplastic, changes, and then unified into cervical intraepithelial neoplasia of three grades by Richart [4-6]. Histopathology continues to drive key decisions on treatment of precancer and invasive cancer; and for this reason has been used to define clinical endpoints for research into cervical screening, HPV vaccines and biomarkers.

Cytopathology forms the basis of cervical screening. The proposal can be traced to the Romanian scientists Aurel Babes and Constantin Daniel in 1927 [7]. In 1941 George Papanicolaou and Herbert Traut created widespread attention with their paper on applying the cervical smear technique to women in the Gynaecology department of New York Hospital in the USA [8].

\section{Histopathology and the concepts of cervical precancer and cancer}

\subsection{Normal cervical histology}

The normal cervix has two distinct epithelial zones: the ectocervix covered by squamous epithelium, and the endocervix lined by simple glandular epithelium [6, 9]. As a result of squamous metaplasia during adolescence and early adult life the endocervical epithelium is replaced by immature squamous epithelium which eventually matures. This squamous metaplastic area is called the transformation zone and is by far the most common site for development of cervical cancer. What makes the transformation zone uniquely sensitive to HPV-induced carcinogenesis is not well understood. It may be a consequence of exposure of actively proliferating stem cells or transit amplifying cells in the basal layer to HPV, specific molecular properties of the immature metaplastic epithelial cells or the local immune system $[10,11]$.

Underneath the epithelium is a layer of connective tissue and beneath this smooth muscle. There is a local cervical immune system in the epithelium and subepithelial tissue, composed of $\mathrm{T}$ and B lymphocytes, plasma cells, NK cells and macrophages and specialized antigen-presenting cells - Langerhans' cells. The local immune system is particularly prominent in certain diseases - for example, lymphoid follicles in follicular cervicitis associated with Chlamydia infection, polymorphs during acute inflammation (eg in gonococcal infection), and the rare condition of eosinophilic cervicitis. Oncogenic HPV, specifically HPV 16 can inhibit activation of the local immune system, mani- fested as a reduction in numbers of epithelial Langerhans' cells $[11,12]$ This may be a key mechanism of viral persistence. All the different immune cells and other specialized cells such as endocervical cells showing tubal metaplasia may express factors considered as potential biomarkers of carcinogenesis and their presence in clinical studies compared to simple laboratory models complicates the application of biomarkers in clinical practice.

\section{The CIN classification}

Carcinoma-in-situ (CIS) has long been recognized as the immediate precursor of invasive cancer, and both squamous CIS and glandular, adenocarcinoma-in-situ (AIS) are recognised. The majority of cervical precancer, like invasive cancer (ICC), shows a squamous differentiation. The cervical intraepithelial neoplasia (CIN) classification system was developed to embrace all potential squamous precancerous lesions and to classify the lesions into grades CIN 1, CIN 2 and CIN 3 [13]. The system is descriptive, the morphological changes are regarded as a continuum, and the three grades are defined by arbitrary criteria, the most important of which is the proportion of the epithelium occupied by undifferentiated, basal type cells (Fig. 1). CIS was grouped with severe dysplasia as CIN 3, and mild and moderate grades of dysplastic change reclassified as CIN 1 and CIN 2 respectively. The classification predates and takes no direct account of the oncogenic role or cytopathic effects of different types of HPV. Use of the CIN system is deeply entrenched in clinical practice and provides for a level of continuity in terminology for diagnostic records $[6,9,14,15]$.

An important modification was introduced in 1988 and revised in 2001 [16,17]. The Bethesda system was started as an attempt to define morphological correlates for the different effects of oncogenic and low-risk HPV in cervical lesions, unify morphology of cervical HPV infection and precancer and create a single terminology for histopathology and cytopathology of precancer. For histology two grades of cervical lesion, LSIL and HSIL were defined (Fig. 1). Neither category precisely relates to the presence of low-risk or of oncogenic HPV, as there are no consistent morphological differences between oncogenic and non-oncogenic HPV infection apart from those of progressive neoplastic transformation recorded in CIN. Each category includes a huge range of lesions. In practice LSIL has been used to correspond to CIN 1, with, variably, some, more mi- 


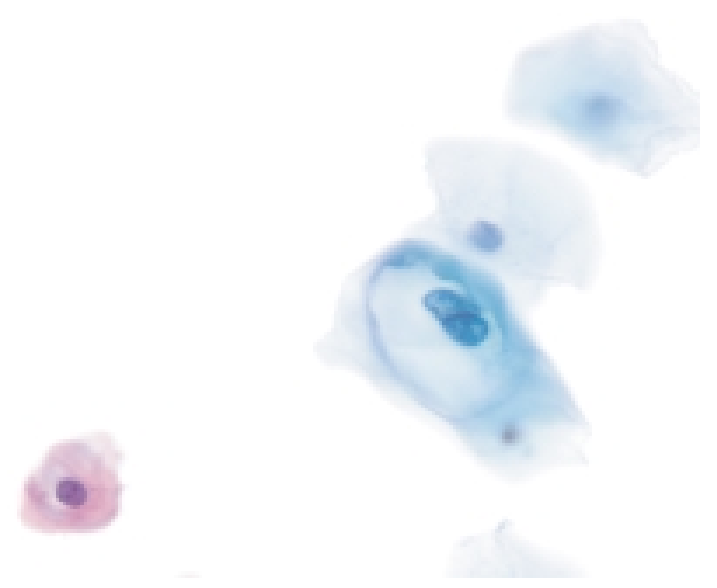

Fig. 1. Schematic representation shows the CIN classification and the Bethesda system, together with the role of HPV infection and persistence. The approximate time to development of lesions estimated from cohort and screening studies is also shown.

nor changes of productive HPV infection. HSIL combines CIN2 and CIN 3 into a single category, covering a very wide range of lesions differing widely in morphological, viral and natural history characteristics. The Bethesda system for cytology has, however, proved popular for clinical use [18].

\subsection{CIN 1}

The characteristics used to define CIN 1 are, firstly, a degree of epithelial hyperplasia, seen as an increase in thickness of the basal and parabasal layers, and secondly, features of HPV replication (productive infection) such as koilocytosis and altered keratinisation, particularly hyperkeratosis and dyskeratosis (Figs 1 and 2). In addition pathologists demand varying degrees of nuclear abnormality for a lesion to qualify as CIN $1[6,9$, $15]$.

Koilocytosis is the most distinctive feature which is recognized in superficial cells by the wrinkled nuclear outline, with nuclear enlargement and hyperchromasia and a sharply etched perinuclear clearing of the cytoplasm. It is the simplest criterion for productive HPV infection to recognize, but is usually very focal and varies greatly in severity and extent, making it a relatively insensitive criterion for HPV infection. Other changes such as basal cell hyperplasia, multinucleate cells, individual cell keratinisation, parakeratosis and papillomatosis are more widespread and are sensitive but not specific features for HPV infection.

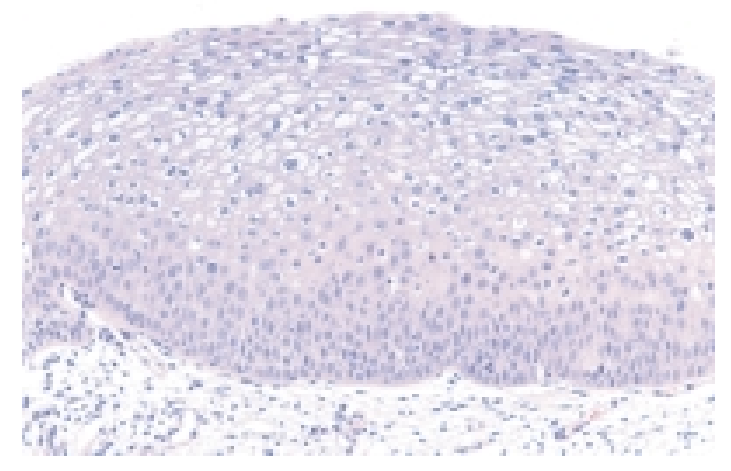

Fig. 2. CIN 1 on cervical biopsy (x20 original magnification) showing hyperplasia of the basal layers (less than one-third of the epithelial thickness) and widespread koilocytosis (vacuolated cells) in the upper layers, together with some binucleate cells. This "typical" case would be accepted by most pathologists as CIN 1, but in other lesions there is widespread variation in the extent and severity of these changes, leading to frequent disagreement on grading.

Pathologists have attempted for over twenty years to identify oncogenic changes by conventional morphology from those of a simple productive HPV infection. Progressive and non-progressive infections are not reproducibly distinguishable $[19,20]$ and there is no general agreement about a threshold of histological abnormality distinguishing progressive or persistent precancerous changes within CIN 1 from transient productive infection. The recent suggestion that CIN 1 should be considered to represent simply the morphological manifestations of productive HPV infection is a significant development supported by studies of expression of HPV oncogenes in CIN [21]. However, by convention many pathologists exclude from CIN 1 a whole range of minor morphological features typical of productive HPV infection. This widespread, but unreproducible practice has not been shown to be of predictive value [20,22]. Attempts to include all HPV related changes in a single category of 'low-grade squamous intra-epithelial abnormalities' has led to over-treatment of frequently regressing lesions in very young women $[23,24]$. Biological markers may represent a solution to this dilemma. Persistence of oncogenic HPV infection over time can distinguish an increased risk of progression to CIN 3 or ICC and the additional expression of the molecular marker p16 can further indicate increased risk of progression [25].

\subsection{CIN 2}

The diagnosis of CIN 2 is important clinically as it represents what is considered to be a transforming le- 


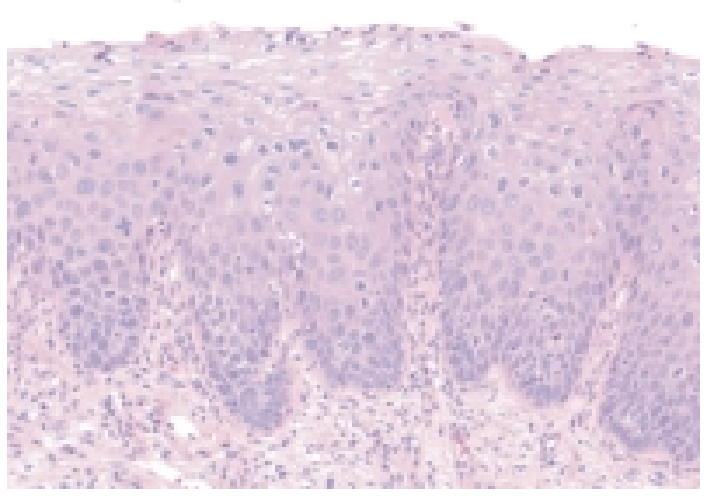

Fig. 3. CIN 2 (x20 original magnification) showing more marked basal hyperplasia (between one- and two-thirds of the epithelial thickness) than CIN 1 with also koilocytosis as in CIN 1. In addition nuclei are enlarged and there are more frequent mitoses and abnormal, multipolar and fragmented mitoses indicating also a high-grade lesion. As in CIN 1 the range of appearances included in CIN 2 leads to disagreement on grading.

sion and the threshold for treatment in most cervical screening programmes. The distinction of CIN 2 from CIN 1 is based on a number of different criteria. The nuclear atypia is more severe than CIN 1; immature proliferating cells persist up to the middle third of the epithelium where cytoplasmic maturation commences. Aneuploidy - and related histological features important in discrimination of CIN1 from CIN 2+, particularly abnormal mitoses and mitoses at a high level in the epithelium, koilocytosis and other changes of productive HPV infection remain a common feature, with frequent bizarre koilocytes and apoptotic or dyskeratotic cells (Fig. 3). The limitations of this category in relation to reproducibility and natural history are discussed below.

\subsection{CIN 3}

CIN 3 is characterized by major loss of orderly maturation of the epithelium with the deepest two-thirds or more of the epithelium replaced by immature abnormal cells with abnormal nuclei and atypical mitoses (Figs 1 and 4). In contrast to the strict definition of carcinomain-situ in which the entire epithelium is replaced by such cells, in CIN 3 there may be some flattening and cytoplasmic maturation of surface squamous cells in the upper third of the epithelium, although these cells are abnormal. CIN 3 is considered the HPV-related, immediate intraepithelial precursor of invasive cervical cancer. Lesions with CIN 3 may have a complex architecture, with both high-grade and low-grade com-

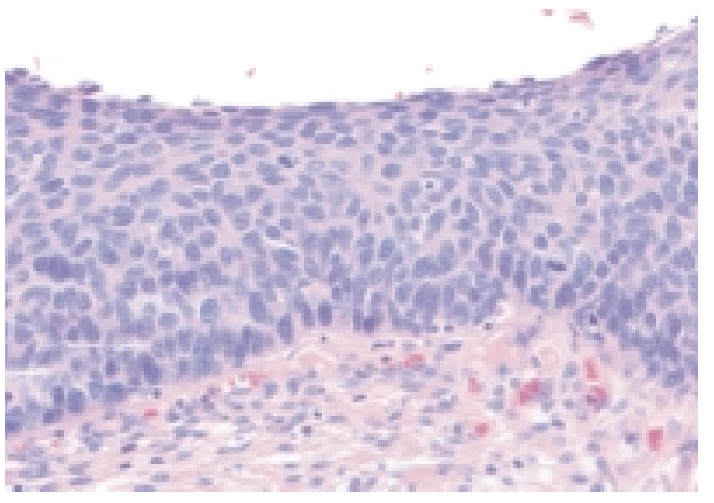

Fig. 4. CIN 3 (x20 original magnification) shows abnormal cells with hyperchromatic (dark) nuclei almost filling the cells occupying almost the entire epithelium. When the entire epithelium is replaced by uniform immature cells it is called carcinoma-in-situ, but in this example there is some flattening and differentiation of cells at the surface.

ponents. These have a definite arrangement with a centripetal structure in which CIN 3 is a central core at the inner part of the transformation zone and is surrounded by CIN 1. This complex architecture may affect the expression of molecular markers eg HPV viral load detection in cervical samples.

There is important heterogeneity within CIN 3, especially in lesion size which is not always routinely recorded. This varies widely in extent of involvement of surface epithelium by at least two orders of magnitude and also in depth of extension into cervical crypts [26-29]. Large lesion size has been correlated with cytological grade of abnormality detected in cytological screening, with poor response to local treatment, and very importantly with early invasive or micro-invasive cancer $[26,29,30]$. As such it may be a reflection of duration and growth of the lesion over many years and may be associated with molecular evidence of accumulating genetic abnormality leading to development of invasive cancer. Other phenotypic changes such as central dyskeratotic necrosis and intralesional squamous maturation are also seen in CIN 3 associated with early invasive cancer [30]. The size of a CIN 3 lesion should be considered in the evaluation of molecular markers for evolution of cervical precancer to cancer.

\subsection{Precursors of adenocarcinoma}

Adenocarcinoma is thought to arise also through a parallel pathway of cervical glandular intraepithelial neoplasia which is closely associated with squamous CIN [15,31]. The association of adenocarcinoma and 
oncogenic HPV is as strong as that of squamous carcinoma, although HPV 18 plays a major role and HPV 16 and 18 together are found in more than $80 \%$ of cervical adenocarcinoma [10].

The natural history of cervical glandular intraepithelial neoplasia is less well understood than that of squamous intraepithelial neoplasia, with which it is frequently associated. It generally affects the transformation zone, but can also involve endocervical crypts and may be discontinuous and multifocal. It is recognized by a combination of architectural changes in glandular organization and cytological abnormalities including cellular stratification, large and irregular nuclei, prominent nucleoli and mitoses. High-grade glandular precancer (adenocarcinoma-in-situ, sometimes called CGIN 3) is relatively well-defined, although it may sometimes be difficult to distinguish from early invasive adenocarcinoma. Low-grade changes (glandular atypia) are more difficult to define, and their outcomes are difficult to predict. These changes are sampled less effectively by conventional cervical cytology, and screening has had less impact on adenocarcinoma than on squamous carcinoma [32] .

\subsection{Invasive cervical cancer}

The morphology, spread and classification of invasive cervical cancer, stage and grade is described well in standard texts of gynaecological pathology and oncology $[31,33,34]$. Over $70 \%$ of invasive cervical cancer is squamous cell carcinoma with adenocarcinoma and adenosquamous carcinomas making up the majority of the rest, together with infrequent small-cell undifferentiated carcinomas and clear cell carcinomas. The last is associated with vaginal adenosis and prenatal exposure to diethylstilboestrol, but, otherwise, all or almost all cervical cancer is considered as due to oncogenic HPV [35]. The rare verrucous carcinoma may be associated with HPV 6 \& 11 [36].

The histological types of squamous carcinoma provide little prognostic information. Small-cell undifferentiated carcinoma shows features of neuro-endocrine epithelial differentiation, and has a less favorable prognosis with surgery. Adenocarcinomas have more positive lymph nodes than squamous carcinomas. The International Federation of Gynecology and Obstetrics (FIGO) staging, based on pathological examination of resected tissue is the basis of determining which approach is used in individual patients in current therapy.

One important category is micro-invasive carcinoma [37-39]. This group of cancers is well defined for squamous carcinoma, but less clear for adenocarcinoma. It constitutes a precisely defined group within the FIGO staging that is usually detected through screening and has very limited superficial invasion only. By setting very precise histological definitions it is possible to identify a group of women with invasive cancer who can be safely managed by local excision.

\subsection{Areas of diagnostic difficulty}

There are some lesions which are unrelated to HPV or neoplasia in both squamous and glandular epithelium that can cause difficulties and errors in pathological diagnosis. These are problems where biomarkers could be of great value in diagnosis, but also create issues for assessment of biomarkers, as expression of biomarkers may differ physiologically from that of the normal squamous or glandular epithelium in these states. In squamous epithelium some of these changes are: basal cell hyperplasia, immature squamous metaplasia, lowoestrogen states and transitional cell metaplasia. In diagnosing glandular precancer there can be problems with lesions of endometriosis and tubo-endometrioid metaplasia, progestogen-associated changes, inflammatory changes in glandular epithelium, Gartner's duct remnants, endocervical polyps, Arias Stella change and microglandular hyperplasia [40-42].

\section{Cytopathology of cervical precancer and cancer}

A number of systems have been employed to attempt to classify the visible changes in cervical smears in a way that is reliable and clinically relevant to the management of screen-detected abnormalities. All systems are based on detection in cervical smears of relatively small numbers of cells showing cytological features of productive HPV infection and of the different grades of histological precancer. How these changes are grouped has been the subject of much controversy, reflecting the difficulty of matching cellular morphology of the individual cells to histological diagnosis and then to treatment. Some of the major systems are shown in Table 1.

\section{Bethesda classification system}

The Bethesda classification is the major current system of terminology providing a standardized format for reporting cervical cytology $[7,17,18]$ (see Table). 
Table 1

Terminology used in reports for cytological and histological tests (Source: IARC 2005b)

\begin{tabular}{|c|c|c|c|c|c|}
\hline \multicolumn{2}{|c|}{ Papanicolaou System } & \multirow{2}{*}{$\begin{array}{l}\text { World Health } \\
\text { Organization }\end{array}$} & \multirow[t]{2}{*}{ CIN } & \multirow{2}{*}{$\begin{array}{l}\text { Bethesda } \\
\text { System }\end{array}$} & \multirow{2}{*}{$\begin{array}{l}\text { UK classification } \\
\text { system }\end{array}$} \\
\hline Grade & Description & & & & \\
\hline Cytological test & & $\begin{array}{l}\text { Cytological and } \\
\text { histological test }\end{array}$ & Histological test & $\begin{array}{l}\text { Cytological and } \\
\text { histological test }\end{array}$ & Cytological \\
\hline I & $\begin{array}{l}\text { Absence of atypical } \\
\text { or abnormal cells }\end{array}$ & & & $\begin{array}{l}\text { Within normal } \\
\text { limits }\end{array}$ & Normal \\
\hline II & $\begin{array}{l}\text { Atypical cytology, } \\
\text { but no evidence } \\
\text { for malignancy }\end{array}$ & & & $\begin{array}{l}\text { Benign cellular } \\
\text { changes } \\
\text { ASC-US/ASC-H }\end{array}$ & Dyskaryosis \\
\hline \multirow[t]{3}{*}{ III } & Cytology suggestive & Mild dysplasia & CIN1 & Low-grade SIL & Mild dyskaryosis \\
\hline & of, but not & Moderate dysplasia & CIN2 & High-grade SIL & Moderately \\
\hline & $\begin{array}{l}\text { conclusive for, } \\
\text { malignancy }\end{array}$ & Severe dysplasia & CIN3 & & dyskaryosis \\
\hline IV & $\begin{array}{l}\text { Cytology strongly } \\
\text { suggestive of } \\
\text { malignancy }\end{array}$ & Carcinoma in situ & CIN3 & & $\begin{array}{l}\text { Severe dyskaryosis } \\
\text { /invasive carcinoma? }\end{array}$ \\
\hline V & $\begin{array}{l}\text { Cytology } \\
\text { conclusive for } \\
\text { malignancy }\end{array}$ & $\begin{array}{l}\text { Microinvasive } \\
\text { carcinoma } \\
\text { Invasive carcinoma }\end{array}$ & $\begin{array}{l}\text { Invasive } \\
\text { carcinoma }\end{array}$ & Invasive carcinoma & $\begin{array}{l}\text { Glandular } \\
\text { neoplasia }\end{array}$ \\
\hline
\end{tabular}

Fig. 5. A group of cells shows changes classified as ASCUS (x60 original magnification). These cells are atypical in terms of nuclear enlargement, a binucleate cell, a "stippled" chromatin, and some vacuolation. These changes can be associated with a wide range of underlying pathology, including CIN2/3 and very occasionally cancer, but often no important pathology.

It was originally developed at a workshop in Bethesda in 1988 , and was widely accepted by the relevant professional societies. In 2001, the American Society for Colposcopy and Cervical Pathology produced a revised consensus statement called "the Bethesda 2001 System" [17]. This can be summarised as:

- Squamous cell abnormalities

1. Atypical squamous cells of undetermined significance (ASC)

ASC-US: the commonest abnormality, including a range of minor cellular abnormalities which may or may not be associated with HPV or cervical cancer development (Fig. 5). Wom-

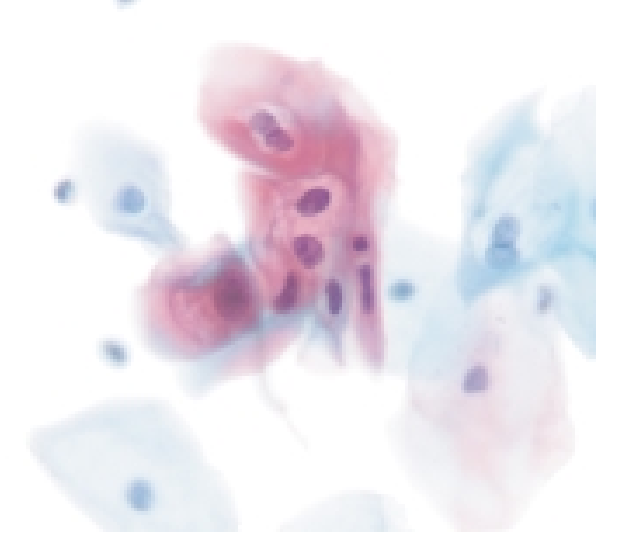

Fig. 6. A group of cells showing changes of LSIL (x60 original magnification). The nuclei are irregular, dark and surrounded by well-defined cytoplasmic vacuolation typical of koilocytes. This picture is relatively reproducible to interpret and associated with productive HPV infection.

en with ASC-US may have findings on cervical examination ranging from negative to invasive cervical cancer.

ASC-H represents morphological abnormalities where the cytopathologist has difficulty in being confident whether the changes are related to a high-grade precancer or not.

2. Squamous intraepithelial lesion (SIL) to denote cervical cancer precursors: Low-grade SIL (LSIL) encompassing: productive HPV changes; mild dysplasia or CIN1 (Fig. 6).

High-grade SIL (HSIL) encompassing: moderate and severe dysplasia CIN2/3 including carcinoma in situ (Fig. 7). 


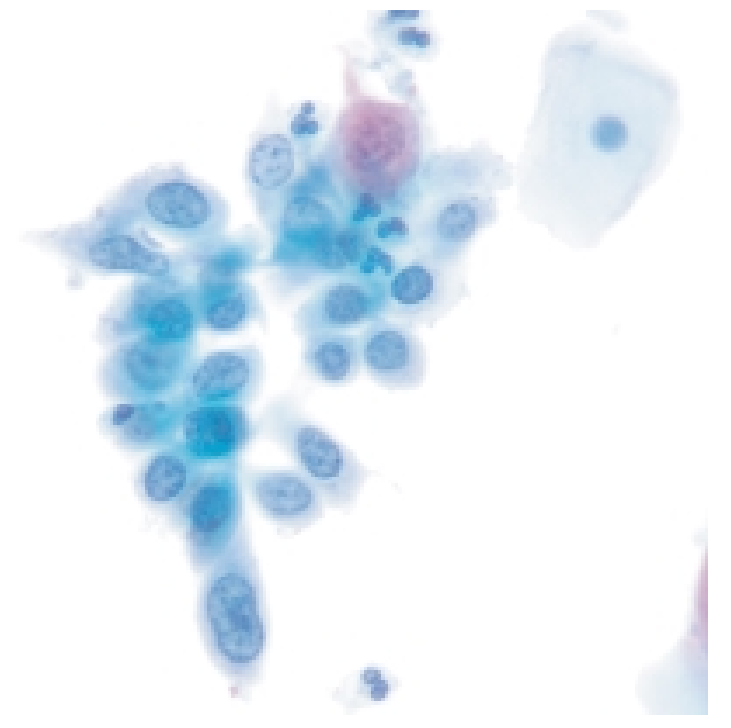

Fig. 7. The changes of HSIL (x60 original magnification) are relatively distinct - a large nucleus with a coarse surrounding membrane and dark blocks of chromatin. They are usually associated with CIN2/3 and cancer.

\section{Squamous cell carcinoma (SCC)}

- Glandular (columnar) cell abnormalities

1. Endometrial cells, cytological benign, in a postmenopausal woman

2. Atypical glandular cells:

Atypical glandular cells (AGC) which may be endocervical, endometrial, or glandular cells not otherwise specified (AGC NOS)

Atypical glandular cells, either endocervical or glandular cells favour neoplasia (AGC favour neoplasia)

Endocervical adenocarcinoma in situ (AIS)

3. Adenocarcinoma: endocervical; endometrial; extrauterine; no obvious site.

While the Bethesda classification system is used in many countries throughout the world, its use is less common in Europe, where many of the organized screening programmes have their own classification schemes (e.g., Finland, the Netherlands and the UK). The terminology used in these setting generally can be translated into the terms in the Bethesda classification, but there is evidence of country-specific differences in interpretation, which makes precise translation more difficult [43].

Before considering the relationship between cytological findings and underlying cervical disease and how this informs the natural history of the progression from cervical HPV infection to invasive cancer it is neces- sary to examine the issues around the performance of cytopathology and histopathology as tests.

\section{Performance of cytopathology and histopathology as tests}

\subsection{Reproducibility}

Many studies have shown that the reproducibility of cervical cytological and histological interpretations is limited. This has important consequences for clinical practice in cervical screening, especially the requirements for regular repeat testing and follow-up investigations and underlies the importance of training and quality control in cervical cytology and histopathology. It also underlies some of the criticism and litigation aimed at cervical screening despite its success in reducing cervical cancer incidence and deaths. The results of the studies of cytological and histological reproducibility are generally consistent. The largest study of both, the ASCUS-LSIL triage study in the USA showed that, overall, the reproducibility of monolayer cytology was moderate $(\mathrm{K}=0.46)$ and equivalent to the reproducibility of punch biopsy and LEEP histopathology. Reproducibility was greatest for high-grade histology, although in this study CIN2 was not separated from CIN 3 and other studies have found CIN 2 to be less reproducible than CIN 3. The lack of reproducibility is generally greatest for less severe interpretations for both cytology and histology, with the interpretation of ASCUS (borderline) cytology and the diagnosis of CIN 1 and CIN 2 histology being the most uncertain diagnoses in most studies [20]. However, the cytological diagnosis of LSIL was found to be reproducible [20]. It is likely that this reproducibility is a consequence of the strict and simple criteria, essentially koilocytosis with nuclear abnormality, required to make the diagnosis.

\subsection{Sensitivity and specificity}

Cytology shows only a modest overall sensitivity but potential high specificity with regard to detection of CIN lesions with a wide range of performance in different laboratories in different countries, and a tradeoff between sensitivity and specificity made differently by different observers. This has been documented in a number of systematic reviews $[44,45]$. A recent overview of European and North American studies on HPV testing in primary cervical cancer screening showed that the sensitivity of cytology for CIN $2+$ was 
$53 \%$ and specificity $96.3 \%$. There was a very wide variation in sensitivity of cytology from $19-77 \%$ between different laboratories in different countries, reinforcing the importance of training and quality control. In the same study HPV DNA testing was confirmed as showing better and more consistent sensitivity (96\%; range 94-97\%) although lower specificity (91\%; range 90-91\%) [46]. This is similar to other reviews $[47,48]$. The lower specificity of HPV DNA testing is affected by the age at which screening by HPV DNA is offered and potentially limits this to women over 25-30 y as well as requiring evidence of persistent infection to avoid increasing the cost and inconvenience of referral for colposcopic follow-up.

\subsection{Improving cytological reproducibility and accuracy}

The varied reproducibility of different cyto-histological diagnostic categories suggests that the complexity of the information on which the diagnosis is based, is important in determining agreement. Training in the use of clearly defined criteria is important and requires support by proficiency testing, performance improvement programs or external quality assurance (EQA), and by internal quality assurance using approaches such as rapid re-screening or random re-screening. Such programmes have been introduced into comprehensive training and QC programmes in the USA and in the UK NHS CSP. Automated prescreening and rescreening using image analysis also may assist in standardisation [49].

There is, however, no evidence that expert pathologists perform significantly better, as opposed to differently, compared to other properly trained specialist pathologists. The ALTS study indeed suggests that the correlation for the expert review group of a more strict diagnosis of CIN 1 with HPV DNA positivity was lower than for the clinical centre pathologists, and did not predict outcome [20].

\subsection{Cytohistological relationships}

The overlapping terminology used in cytological and histological classifications of cervical precancer implies a close relationship between the findings of the two methodologies. This is only an approximation. In particular, low-grade cytology (both ASCUS (borderline) and LSIL (mild dyskaryosis)) can be associated with underlying normality, CIN 1, but also CIN2 and CIN 3, and infrequently invasive cancer. The relation- ship is affected by the subjectivity and limited reproducibility of both tests and colposcopy used to identify lesions for biopsy, and by the sampling process for cytology and biopsy. One important, additional, biological factor that has received only limited attention so far is lesion size, especially for CIN 3 [26-29]. Although there is considerable variation, CIN 1 and CIN 2 lesions are mostly small, with CIN 3 lesions showing a wide range of size. Increasing size of CIN 3 is associated with presence of early invasive cervical cancer and also increases the likelihood of a high-grade cytological abnormality being detected. Small lesions are associated with low-grade cytology and high-grade cytology is associated with large lesions. This is important with regard to the detection of CIN 3 at different ages and to understanding the limitations of knowledge of the natural history of CIN 3.

\section{Natural history of cervical precancer and cancer}

In an unscreened population it has been estimated from mathematical models that the lifetime risk of cervical cancer is about $3-4 \%$, although the rates up to age $65 y$ in developing countries are about $1.5 \%[32,50$, 51]. Understanding the progression of precancer to invasive cancer is based on analysis of data from cervical screening and from cohort studies. In recent years, this has been supplemented by information on HPV type distribution in cytological abnormality and cohort studies of HPV infection and subsequent cervical precancer or cancer.

\subsection{Screening and cohort studies of women with cytohistological abnormalities}

Data from screening studies was reviewed by Oster (1993). This review found? 3529 cases of CIN 1 of which $57 \%$ showed regression, persistence was found in $32 \%$, progression to CIN 3 in $11 \%$ and progression to invasive cancer in $1 \%$ of cases. The corresponding figures for CIN 2 were $43 \%, 35 \%, 22 \%$ and $5 \%$ respectively. Melnikow [52] demonstrated in a meta-analysis of the published literature similar rates of regression of cytological abnormalities: $68 \%(95 \% \mathrm{CI}=58-79 \%)$ of ASC-US cases; $47 \%$ (95\% CI $=36-59 \%)$ of LSIL and $35 \%(95 \% \mathrm{CI}=17-53 \%)$ of HSIL cases. Estimated rates of progression of cytological abnormalities over 24 months range from 7\% for ASCUS to about $21 \%$ for LSIL. Progression to ICC over 24 months was rare: $1.4 \%$ for HSIL and $0.2 \%$ for ASC-US [52]. 
Data from cohort studies indicate that regression of lesions may be very frequent in young women. In one of the few longitudinal studies that included very young women (13-22 y) who were HPV positive at enrolment and had LSIL detected at baseline or during the study $91 \%$ (95\% CI $=84-99 \%$ ) regressed by 36 months [23]. Progression to HSIL occurred in $3 \%$ at this time. In another study of girls 18 years or younger with LSIL, $28 \%$ had regressed by 12 months, $55 \%$ by 24 months and $62 \%$ by 36 months [53].

\subsection{Distribution of HPV types in precancerous lesions}

The above studies take no account of the impact of HPV type on outcome. Studies of prevalence of different HPV types in cervical precancerous lesions and in cancer have been summarized in a number of systematic reviews [54-56]. The total HPV DNA positivity rose from $71.1 \%$ in LSIL to $88.1 \%$ in cancer. Such figures are probably substantial underestimates as they are based on a wide range of studies with varying cytology, histology and HPV detection methodology, including sub-optimal studies. The major types of oncogenic HPV are found in all grades of lesions, with HPV 16 predominating. Importantly the distribution of HPV types changes progressively from low-grade to invasive cancer [56,57] with HPV 16 and 18 being the two types which increase in frequency from low-grade to highgrade lesions (2.0 and 1.5 times respectively). There is a decrease in frequency with other oncogenic types ranging from slight $(0.85)$ for HPV 45 to 0.1 for HPV 53. In addition a single cervix may contain multiple lesions which may be of different HPV types. There is a decrease in frequency of multiple type infections from up to $50 \%$ in low-grade lesions to about $4 \%$ in invasive cancer $[54,55,58]$.

\subsection{Incident and persistent HPV infection and cervical precancer or cancer}

Cohort studies of women uninfected at entry show that incident oncogenic HPV infection is associated with the development of CIN lesions of all grades within 3-24 months. In the case of HPV 16, CIN 1 has been found within 3 months of first detecting infection, and for CIN 2 within 6 months [59,60]. Median time of progression from incident infection to lesions is somewhat longer, around 15-16 months [61,62], although all such estimates are affected by sampling interval and the limited sensitivity of cytology for detect- ing morphological changes compared to very sensitive viral detection. Many of the issues have been reviewed recently $[63,64]$ Studies also show that the risk of highgrade precancer or invasive cancer is increased with increasing duration of persistence of oncogenic HPV infection especially over 12 months or more [65-69]. Persistent type-specific oncogenic HPV infection has been proposed as a more reliable surrogate indicator of cancer risk for screening and trials than the less reproducible, current, treatment threshold of CIN 2 or worse. The risk for women over $30 \mathrm{y}$ with oncogenic HPV of CIN 3 over 10 years of follow-up has been found to be particularly high for certain oncogenic HPVs $-17 \%$ for HPV 16 and 14\% for HPV 18, compared to $3 \%$ for other oncogenic HPV [70] and similar esults have been obtained in other studies [71]. This is consistent with cross-sectional data showing the different distributions of individual oncogenic HPVs between precancers of different grades and cancer.

\subsection{A model of the natural history of cervical carcinogenesis}

Combining information on natural history of cervical precancer from all sources allows proposal of a model going beyond the traditional concept of cervical carcinogenesis proceeding gradually in 10-yearly steps from CIN1 to CIN 2 to CIN 3 over 20-30 years. CIN 1 and CIN 2 can arise within a few months of incident infection and even CIN 3 within 1 year, although the median time for these events is likely to be somewhat longer. Reconciling the cohort studies with the screening data implies there is then likely to be a long period of evolution of high-grade lesions, possibly in transition from CIN 2 to CIN 3, but also probably associated with expansion in size within CIN 3, accompanying multiple genetic and phenotypic changes leading in some women to invasive cancer.

This model leaves open questions which cannot be fully resolved on present evidence. Such uncertainties are important considerations when designing studies of potential biomarkers of precancerous progression, and may be finally resolved by application of markers.

\section{Cervical screening}

Organised cervical cytological screening has been shown to reduce cervical cancer incidence and mortality by $70-90 \%$ [2,72]. Within all major national screening programmes and in the USA where screen- 
ing practice is recommended by the American Society for Colposcopy and Cervical Pathology on the basis of a National Consensus Conference and by other organisations, cervical screening practice is based on regularly updated guidelines. These are, when possible, informed by critical appraisal of evidence from diagnostic studies, from trials and from mathematical modelling of cost-effectiveness. Current screening is based on regular cervical cytology sampling of adult women at intervals of one to five years. About 1 in 15 women screened have some abnormality requiring further examination or treatment. The management of women with equivocal (ASCUS) smears is an important issue, generating anxiety and cost. About $10 \%$ will have CIN2/3 and 1/1000 or less will have cervical cancer. The high sensitivity and hence negative predictive value of HPV DNA testing in the triage situation make this an effective test, and, especially when used as a reflex test on a liquid-based cytology specimen, very efficient. Women who are HPV negative can be reassured and avoid further follow-up. The management of women who are HPV positive is less clear. Immediate colposcopic referral may lead to over-treatment of regressing lesions and will also fail to detect CIN 3 which may develop subsequently [73].

European Union guidelines on cervical screening recommend that cervical cytology should be part of an organised screening programme beginning between 20-30 years and continuing until at least 60 years, with an interval of 3-5 y. In practice there is considerable variation between European countries and even more worldwide. In the USA, although current recommendations support screening at three yearly intervals there is a longstanding, but expensive practice of annual screening starting shortly after commencing sexual activity.

Effectiveness and cost-effectiveness are important issues in screening and very relevant in assessing the likelihood of uptake of a new biomarker. Such issues can be explored by randomised clinical trials (e.g. ALTS) but these use intermediate or surrogate clinical endpoints such as CIN2/3 or CIN3 and any impact on invasive cancer and cost-effectiveness can only be studied prior to widespread clinical implementation by mathematical modelling. Such studies have addressed coverage, interval and age range of screening as well as triage algorithms, HPV DNA testing, liquid-based cytology and, more recently, the potential interaction of cervical screening and prophylactic HPV vaccination. A consistent result of such modelling is that effectiveness of cervical screening is largely driven by coverage of the at- risk population of women and by the frequency of their screening, although increasing this above every three years has little impact. Cost-effectiveness is strongly influenced by the age range over which screening is performed and by the frequency of screening. The performance characteristics of the screening test follow this in importance $[74,75]$. These characteristics are, however, important in assessing alternative diagnostic tests. One example is that the major limitation of HPV DNA as a screening test is that it does not have high specificity and this is influenced by the frequency of incident and prevalent infection in the population [46, 48]. This varies widely geographically as well as with age. In considering screening tests any impact on frequency of screening could well outweigh other limitations in determining cost-effectiveness. Biomarkers are potentially important as primary screening tests, and, importantly, because of their potential higher specificity for high-grade precancer, as a second stage triage test, preferably as a reflex test which can be performed in the laboratory on a primary liquid-based cytology specimen after cytology or HPV DNA screening.

\subsection{Pathology and the assessment of new diagnostic tests for screening and triage}

Unlike therapeutics there is no standard regulatory procedure for diagnostic testing. Evaluating a new biomarker often begins with cross-sectional studies in which the potential biomarker is compared for reproducibility, diagnostic sensitivity and specificity on clinical specimens with an established test such as cytology (or even histopathology itself) in predicting a histopathology outcome, usually CIN. CIN 1 is now seen not so much as a precancerous disease entity, but as a histological marker for productive infection. CIN2 $\& 3$, combined with adenocarcinoma-in-situ has been widely used as a combined endpoint of high-grade precancer in clinical trials for the assessment of diagnostic tests or triage algorithms for women with abnormal smears and in primary cervical screening, and also for phase 3 studies of prophylactic HPV vaccines. Making histopathology endpoints reproducible requires the use of a pathology panel through which a review diagnosis based on independent reading and concurrence of at least two expert observers is used to define the endpoints of the study. This approach has been used in clinical trials. When multiple sections are examined from a single block, the grade of a lesion may change through the block. When it is important to link CIN grade to individual HPV types or to markers using ex- 
tracted DNA or RNA from tissue (as in assessing impact of prophylactic HPV vaccination) it is necessary to link the histology panel diagnosis to a sandwich process of taking sections for DNA or RNA analysis between sections subjected to histological examination.

Demonstration of sensitivity, specificity and reproducibility of a new marker is a basic requirement, but this is not sufficient to ensure uptake and reimbursement as these are also driven by the impact of a new marker on effectiveness and cost-effectiveness. A high sensitivity improves effectiveness and a high specificity improves cost-effectiveness. Neither cervical cytological screening nor HPV DNA testing are perfect by these criteria and programmatic issues such as screening interval and the necessity of triage are heavily influenced by test performance with substantial implications for health care costs and for the women concerned. It is therefore necessary to complement the data on test performance by an exploration in a clinical trial and through mathematical modelling of the true impact of the new test on clinical effectiveness and costeffectiveness. The endpoint of a trial is usually CIN 2 or CIN 3. CIN 2 is the threshold for surgical treatment of CIN, but some experts have a preference for CIN 3 as the natural history of CIN 2 includes acute and regressing lesions. Studies using CIN3 and ICC as endpoints in the context of screening or other cervical cancer prevention are limited by the requirement for large study populations and/or many years, even decades, of follow-up. Therefore potential impact on invasive cancer is usually assessed by mathematical modelling. Review of the current evaluations of HPV DNA testing and of liquid-based cytology provide a guide as to the approaches needed to assess new biomarkers $[46,48$, 64].

\section{Conclusion}

Biomarkers offer exciting opportunities to overcome the limitations of current morphological methods of screening and diagnosis in cervical cancer prevention and cancer management. Understanding the theory and practice of cervical histopathology and cytopathology is important for the assessment of biomarkers. Cytopathology remains the key comparator for evaluating the performance of a biomarker in screening, and histopathology provides the major surrogate endpoints of high-grade precancer - CIN 2 or worse. An important issue for both is standardization of subjective morphological interpretation when used as a comparator or endpoint, requiring careful attention to reproducibility and quality control of the diagnostic processes. Use of multiple readers is essential for key endpoints, and careful attention is also needed to link the histopathology endpoints accurately to their HPV status.

The evaluation of biomarkers for cervical cancer prevention even with standardisation of pathology comparators and endpoints, cannot be considered in isolation from the picture of current clinical and laboratory practices, or from potential future changes in screening practice and other important, new developments such as prophylactic HPV vaccination. Pathology is the key in providing endpoints for assessing diagnostic sensitivity and specificity and for clinical trials. However, in cervical screening and cervical cancer prevention it is effectiveness and cost-effectiveness of cervical cancer prevention that ultimately will determine the use of a new biomarker. The performance data and clinical trial results against surrogate CIN2/3 endpoints are important for providing data for mathematical models to evaluate these rapidly and economically.

A final major issue in planning studies using pathology endpoints is that cervical cancer preventive practice is changing. HPV testing has been introduced widely for triage of ASCUS smears and is under research for primary screening. Most importantly the future impact of prophylactic vaccination against the major types of oncogenic HPV infection, types 16 \& 18 needs to be taken into account. Prophylactic HPV vaccination of uninfected women has been shown to prevent abnormal smears and CIN associated with HPV 16 \& 18 [60,7678] and this protection may extend to other HPV types, in particular HPV 45 and 31 [78]. Effective prophylactic vaccination will alter the natural history of the residual precancer when HPV 16/18 driven lesions are removed. The residual disease will be predominantly associated with other oncogenic and non-oncogenic HPV types. The residual oncogenic HPV lesions are probably less aggressive from cohort studies, and the reduction in frequency of high-grade lesions will decrease the predictive value of abnormal screening results $[73,79]$. This impact on test performance is likely to affect cytology most severely, and, together with the need to identify any true "breakthrough" cases of HPV $16 / 18$ high-grade precancer from other CIN in vaccinated women could drive a change to HPV testing and typing, and the use of biomarkers to improve accuracy. To meet these challenges the assessment of biomarkers against pathology endpoints needs to be done with careful attention to defining pathology endpoints reliably, linking these to HPV status and also with careful 
consideration of the current and future clinical and epidemiological context in which the biomarker is likely to be used.

\section{Acknowledgments}

The author would like to thank Dr. Ron Luff of Quest Diagnostics, Teterboro, NJ, USA, for providing the photomicrographs.

\section{References}

[1] M.E. van den Akker-van Marle, M. van Ballegooijen, G.J. van Oortmarssen, R. Boer and J.D. Habbema, Cost-effectiveness of cervical cancer screening: comparison of screening policies, J Natl Cancer Inst 94 (2002), 193-204.

[2] A. Anttila, G. Ronco, G. Clifford, F. Bray, M. Hakama, M. Arbyn et al., Cervical cancer screening programmes and policies in 18 European countries, Br J Cancer 91 (2004), 935-941.

[3] J. Peto, C. Gilham, O. Fletcher and F.E. Matthews, The cervical cancer epidemic that screening has prevented in the UK, Lancet 364 (2004), 249-256.

[4] R.M. Richart, Natural history of cervical intraeithelial neoplasia, Clin Obstet Gynecol (1968), 5.

[5] R.M. Richart, Cervical intraepithelial neoplasia, Pathol Annu 8 (1973), 301-328.

[6] B. Anderson, Premalignant and malignant squamous lesions of the cervix, in: Haines and Taylor: Obstetrical and Gynaecological Pathology, H. Fox, ed., 1995, pp. 273-322.

[7] P. Virtej and C. Vasiliu, Cytodiagnosis in cervical neoplasia: from the Babes/Papanicolaou smear to the actual Bethesda System, Clin Exp Obstet Gynecol 30 (2003), 173-177.

[8] G.N. Papanicolaou and H.F. Traut, The diagnostic value of vaginal smears in carcinoma of the uterus. 1941, Arch Pathol Lab Med 121 (1997), 211-224.

[9] M.C. Anderson, C.L. Brown, C.H. Buckley, H. Fox, D. Jenkins, D.G. Lowe et al., Current views on cervical intraepithelial neoplasia, J Clin Pathol 44 (1991), 969-978.

[10] N. Munoz, X. Castellsague, A.B. de Gonzalez and L. Gissmann, Chapter 1: HPV in the etiology of human cancer, Vaccine 24 (2006), S3:S1-S10.

[11] M. Stanley, Immune responses to human papillomavirus, Vaccine (2005).

[12] S.K. Tay, D. Jenkins, P. Maddox, M. Campion and A. Singer, Subpopulations of Langerhans' cells in cervical neoplasia, $\mathrm{Br}$ J Obstet Gynaecol 94 (1987), 10-15.

[13] Histopathology Reporting in Cervical Screening, Ed. Fox H \& Buckley CH. NHSCSP Publication 10, 1999, Sheffield ISBN 1871997682.

[14] D. Jenkins, The pathology of lower genital tract premalignancy, Clin Pract Gynaecol (1987).

[15] M. Stoler, The pathology of cervical neoplasia, Kluwer Academic Publishers, 2004.

[16] D. Solomon, The 1988 Bethesda system for reporting cervical/vaginal cytological diagnoses, Acta Cytologica (1989).

[17] D. Solomon, D. Davey, R. Kurman, A. Moriarty, D. O'Connor, M. Prey et al., The 2001 Bethesda System: terminology for reporting results of cervical cytology, JAMA 287 (2002), 21142119.
[18] C. Bergeron, The 2001 Bethesda system, Salud Publica Mex 45(Suppl 3) (2003), S340-S344.

19] D. Jenkins, S.K. Tay, D.J. McCance, M.J. Campion, P.K. Clarkson and A. Singer, Histological and immunocytochemical study of cervical intraepithelial neoplasia (CIN) with associated HPV 6 and HPV 16 infections, J Clin Pathol 39 (1986), 1177-1180.

[20] M.H. Stoler and M. Schiffman, Interobserver reproducibility of cervical cytologic and histologic interpretations: realistic estimates from the ASCUS-LSIL Triage Study, JAMA 285 (2001), 1500-1505.

[21] K. Middleton, W. Peh, S. Southern, H. Griffin, K. Sotlar, T. Nakahara et al., Organization of human papillomavirus productive cycle during neoplastic progression provides a basis for selection of diagnostic markers, J Virol 77 (2003), 1018610201.

[22] J.T. Cox, M. Schiffman and D. Solomon, Prospective followup suggests similar risk of subsequent cervical intraepithelial neoplasia grade 2 or 3 among women with cervical intraepithelial neoplasia grade 1 or negative colposcopy and directed biopsy, Am J Obstet Gynecol 188 (2003), 1406-1412.

[23] A.B. Moscicki, S. Shiboski, N.K. Hills, K.J. Powell, N. Jay, E.N. Hanson et al., Regression of low-grade squamous intraepithelial lesions in young women, Lancet 364 (2004), 16781683.

[24] A. Szarewski and P. Sasieni, Cervical screening in adolescents-at least do no harm, Lancet 364 (2004), 16421644.

[25] S.S. Wang, M. Trunk, M. Schiffman, R. Herrero, M.E. Sherman, R.D. Burk et al., Validation of p16INK4a as a marker of oncogenic human papillomavirus infection in cervical biopsies from a population-based cohort in Costa Rica, Cancer Epidemiol Biomarkers Prev 13 (2004), 1355-1360.

[26] P. Tidbury, A. Singer and D. Jenkins, CIN 3: the role of lesion size in invasion, Br J Obstet Gynaecol 99 (1992), 583-586.

[27] M.E. Sherman, S.S. Wang, R. Tarone, L. Rich and M. Schiffman, Histopathologic extent of cervical intraepithelial neoplasia 3 lesions in the atypical squamous cells of undetermined significance low-grade squamous intraepithelial lesion triage study: implications for subject safety and lead-time bias, Cancer Epidemiol Biomarkers Prev 12 (2003), 372-379.

[28] M.E. Sherman, S.S. Wang, C.M. Wheeler, L. Rich, P.E. Gravitt, R. Tarone et al., Determinants of human papillomavirus load among women with histological cervical intraepithelial neoplasia 3: dominant impact of surrounding lowgrade lesions, Cancer Epidemiol Biomarkers Prev 12 (2003), 1038-1044.

[29] M.R. Jarmulowicz, D. Jenkins, S.E. Barton, A.L. Goodall, A. Hollingworth and A. Singer, Cytological status and lesion size: a further dimension in cervical intraepithelial neoplasia, Br J Obstet Gynaecol 96 (1989), 1061-1066.

[30] A.I. al Nafussi and D.E. Hughes, Histological features of CIN3 and their value in predicting invasive microinvasive squamous carcinoma, J Clin Pathol 47 (1994), 799-804.

[31] N.R. Griffin and M. Wells, Premalignant and malignant glandular lesions of the cervix, Edinburgh, Churchill Livingstone, 1995.

[32] D.M. Parkin and F. Bray, Chapter 2: The burden of HPVrelated cancers, Vaccine 24(Suppl 3) (2006), S11-S25.

[33] C.H. Buckley, Tumors of the cervix uteri, Edinburgh, Churchill Livingstone, 1995.

[34] J.H. Shepherd, Revised FIGO staging for gynaecological cancer, Br J Obstet Gynaecol 96 (1989), 889-892. 
[35] J.M. Walboomers, M.V. Jacobs, M.M. Manos, F.X. Bosch, J.A. Kummer, K.V. Shah et al., Human papillomavirus is a necessary cause of invasive cervical cancer worldwide, J Pathol 189 (1999), 12-19.

[36] C.J. Lacey, C.M. Lowndes and K.V. Shah, Chapter 4: Burden and management of non-cancerous HPV-related conditions: HPV-6/11 disease, Vaccine 24(Suppl 3) (2006), S35-S41.

[37] E. Burghardt, F. Girardi, M. Lahousen, H. Pickel and K. Tamussino, Microinvasive carcinoma of the uterine cervix (International Federation of Gynecology and Obstetrics Stage IA), Cancer 67 (1991), 1037-1045.

[38] N. Tsukamoto, T. Kaku, K. Matsukuma, T. Matsuyama, T. Kamura, T. Saito et al., The problem of stage Ia (FIGO, 1985) carcinoma of the uterine cervix, Gynecol Oncol 34 (1989), $1-6$.

[39] F. Girardi et al., Small FIGO stage Ib cervical cancer, Gynecologic Oncology (1994).

[40] S.M. Ismail, Cone biopsy causes cervical endometriosis and tubo-endometrioid metaplasia, Histopathology 18 (1991), 107-114.

[41] A. al Nafussi and M. Rahilly, The prevalence of tubo-endometrial metaplasia and adenomatoid proliferation, Histopathology 22 (1993), 177-179.

[42] J.A. Ferry and R.E. Scully, Mesonephric remnants, hyperplasia, and neoplasia in the uterine cervix. A study of 49 cases, Am J Surg Pathol 14 (1990), 1100-1111.

[43] D.R. Scott, B. Hagmar, P. Maddox, A. Hjerpe, J. Dillner, J. Cuzick et al., Use of human papillomavirus DNA testing to compare equivocal cervical cytologic interpretations in the United States, Scandinavia, and the United Kingdom, Cancer 96 (2002), 14-20.

[44] M.T. Fahey, L. Irwig and P. Macaskill, Meta-analysis of Pap test accuracy, Am J Epidemiol 141 (1995), 680-689.

[45] K. Nanda, D.C. McCrory, E.R. Myers, L.A. Bastian, V. Hasselblad, J.D. Hickey et al., Accuracy of the Papanicolaou test in screening for and follow-up of cervical cytologic abnormalities: a systematic review, Ann Intern Med 132 (2000), 810-819.

[46] J. Cuzick, M.H. Mayrand, G. Ronco, P. Snijders and J. Wardle, Chapter 10: New dimensions in cervical cancer screening, Vaccine 24(Suppl 3) (2006), S90-S97.

[47] M. Arbyn, F. Buntinx, M. Van Ranst, E. Paraskevaidis, P. Martin-Hirsch and J. Dillner, Virologic versus cytologic triage of women with equivocal Pap smears: a meta-analysis of the accuracy to detect high-grade intraepithelial neoplasia, $J$ Natl Cancer Inst 96 (2004), 280-293.

[48] M. Arbyn, P. Sasieni, C.J. Meijer, C. Clavel, G. Koliopoulos and J. Dillner, Chapter 9: Clinical applications of HPV testing: A summary of meta-analyses, Vaccine 24(Suppl 3) (2006), S78-S89.

[49] M.E. Sherman, M. Schiffman, R. Herrero, D. Kelly, C. Bratti, L.J. Mango et al., Performance of a semiautomated Papanicolaou smear screening system: results of a population-based study conducted in Guanacaste, Costa Rica, Cancer 84 (1998), 273-280.

[50] S.J. Goldie, D. Grima, M. Kohli, T.C. Wright, M. Weinstein and E. Franco, A comprehensive natural history model of HPV infection and cervical cancer to estimate the clinical impact of a prophylactic HPV-16/18 vaccine, Int J Cancer 106 (2003), 896-904.

[51] S.J. Goldie, M. Kohli, D. Grima, M.C. Weinstein, T.C. Wright, F.X. Bosch et al., Projected clinical benefits and costeffectiveness of a human papillomavirus $16 / 18$ vaccine, $J$ Natl Cancer Inst 96 (2004), 604-615.
[52] J. Melnikow, J. Nuovo, A.R. Willan, B.K. Chan and L.P. Howell, Natural history of cervical squamous intraepithelial lesions: a meta-analysis, Obstet Gynecol 92 (1998), 727-735.

[53] J.D. Wright, R.M. Davila, K.R. Pinto, D.F. Merritt, R.K. Gibb, J.S. Rader et al., Cervical dysplasia in adolescents, Obstet Gynecol 106 (2005), 115-120.

[54] G.M. Clifford, J.S. Smith, M. Plummer, N. Muñoz and S. Franceschi, Human papillomavirus types in invasive cervical cancer worldwide: a meta-analysis, Br J Cancer 88 (2003), 63-73.

[55] G.M. Clifford, J.S. Smith, T. Aguado and S. Franceschi, Comparison of HPV type distribution in high-grade cervical lesions and cervical cancer: a meta-analysis, Br J Cancer 89 (2003), 101-105.

[56] G. Clifford, S. Franceschi, M. Diaz, N. Munoz and L.L. Villa, Chapter 3: HPV type-distribution in women with and without cervical neoplastic diseases, Vaccine 24(Suppl 3) (2006), S26S34.

[57] S. Franceschi and G.M. Clifford, Re: A study of the impact of adding HPV types to cervical cancer screening and triage tests, J Natl Cancer Inst 97 (2005), 938-939.

[58] G.M. Clifford, R.K. Rana, S. Franceschi, J.S. Smith, G. Gough and J.M. Pimenta, Human papillomavirus genotype distribution in low-grade cervical lesions: comparison by geographic region and with cervical cancer, Cancer Epidemiol Biomarkers Prev 14 (2005), 1157-1164.

[59] L.A. Koutsky, K.K. Holmes, C.W. Critchlow, C.E. Stevens, J. Paavonen, A.M. Beckmann et al., A cohort study of the risk of cervical intraepithelial neoplasia grade 2 or 3 in relation to papillomavirus infection, N Engl J Med 327 (1992), 12721278.

[60] D.M. Harper, E.L. Franco, C. Wheeler, D.G. Ferris, D. Jenkins, A. Schuind et al., Efficacy of a bivalent L1 virus-like particle vaccine in prevention of infection with human papillomavirus types 16 and 18 in young women: a randomised controlled trial, Lancet 364 (2004), 1757-1765.

[61] R.L. Winer, N.B. Kiviat, J.P. Hughes, D.E. Adam, S.K. Lee, J.M. Kuypers et al., Development and duration of human papillomavirus lesions, after initial infection, J Infect Dis 191 (2005), 731-738.

[62] H. Trottier, S. Mahmud, L. Lindsay, D. Jenkins, S. Wieting and E. Franco, Natural history of HPV infection and cervical lesions in women from the placebo arm of an AS04 adjuvanted HPV-16/18 L1 vaccine trial. EUROGIN.

[63] A.B. Moscicki, M. Schiffman, S. Kjaer and L.L. Villa, Chapter 5: Updating the natural history of HPV and anogenital cancer, Vaccine 24(Supp1 3) (2006), S42-S51.

[64] H.C. Kitchener, P.E. Castle and J.T. Cox, Chapter 7: Achievements and limitations of cervical cytology screening, Vaccine 24(Suppl 3) (2006), S63-S70.

[65] J.P. Bory, J. Cucherousset, M. Lorenzato, R. Gabriel, C. Quereux, P. Birembaut et al., Recurrent human papillomavirus infection detected with the hybrid capture II assay selects women with normal cervical smears at risk for developing high grade cervical lesions: a longitudinal study of 3,091 women, Int J Cancer 102 (2002), 519-525.

[66] V. Dalstein, D. Riethmuller, J.L. Pretet, C.K. Le Bail, J.L. Sautiere, J.P. Carbillet et al., Persistence and load of high-risk HPV are predictors for development of high-grade cervical lesions: a longitudinal French cohort study, Int J Cancer 106 (2003), 396-403.

[67] N.F. Schlecht, S. Kulaga, J. Robitaille, S. Ferreira, M. Santos, R.A. Miyamura et al., Persistent human papillomavirus infec- 
tion as a predictor of cervical intraepithelial neoplasia, JAMA 286 (2001), 3106-3114.

[68] G.Y. Ho, R.D. Burk, S. Klein, A.S. Kadish, C.J. Chang, P. Palan et al., Persistent genital human papillomavirus infection as a risk factor for persistent cervical dysplasia, J Natl Cancer Inst 87 (1995), 1365-1371.

[69] K.L. Wallin, F. Wiklund, T. Angstrom, F. Bergman, U. Stendahl, G. Wadell et al., Type-specific persistence of human papillomavirus DNA before the development of invasive cervical cancer, N Engl J Med 341 (1999), 1633-1638.

[70] M.J. Khan, P.E. Castle, A.T. Lorincz, S. Wacholder, M. Sherman, D.R. Scott et al., The elevated 10-year risk of cervical precancer and cancer in women with human papillomavirus (HPV) type 16 or 18 and the possible utility of type-specific HPV testing in clinical practice, J Natl Cancer Inst 97 (2005), 1072-1079.

[71] P.E. Castle, D. Solomon, M. Schiffman and C.M. Wheeler, Human papillomavirus type 16 infections and 2-year absolute risk of cervical precancer in women with equivocal or mild cytologic abnormalities, J Natl Cancer Inst 97 (2005), 10661071.

[72] M. Hicks and M. Piver, How to obtain an accurate pap smear, Med Asp Human Sexuality (1991).

[73] D. Jenkins, Public Health issues related to HPV vaccination, in: Emerging issues on HPV infections from science to practice, J. Monsonego, ed., Karger, 2006.
[74] S.J. Goldie, J.D. Goldhaber-Fiebert and G.P. Garnett, Chapter 18: Public health policy for cervical cancer prevention: The role of decision science, economic evaluation, and mathematical modeling, Vaccine 24(Suppl 3) (2006), S155-S163.

[75] S.J. Goldie, J.J. Kim and E. Myers, Chapter 19: Costeffectiveness of cervical cancer screening, Vaccine 24(Suppl 3) (2006), S164-S170.

[76] L.A. Koutsky, K.A. Ault, C.M. Wheeler, D.R. Brown, E. Barr, F.B. Alvarez et al., A controlled trial of a human papillomavirus type 16 vaccine, N Engl J Med 347 (2002), 16451651.

77] C. Mao, L.A. Koutsky, K.A. Ault, C.M. Wheeler, D.R. Brown, D.J. Wiley et al., Efficacy of human papillomavirus-16 vaccine to prevent cervical intraepithelial neoplasia: a randomized controlled trial, Obstet Gynecol 107 (2006), 18-27.

[78] D.M. Harper, E.L. Franco, C.M. Wheeler, A.B. Moscicki, B. Romanowski, C.M. Roteli-Martins et al., Sustained efficacy up to 4.5 years of a bivalent $\mathrm{L} 1$ virus-like particle vaccine against human papillomavirus types 16 and 18: follow-up from a randomised control trial, Lancet 367 (2006), 1247-1255.

[79] E.L. Franco, J. Cuzick, A. Hildesheim and S. de Sanjose, Chapter 20: Issues in planning cervical cancer screening in the era of HPV vaccination, Vaccine 24(Suppl 3) (2006), S171S177. 


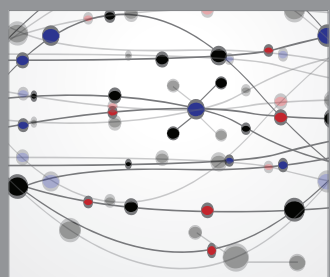

The Scientific World Journal
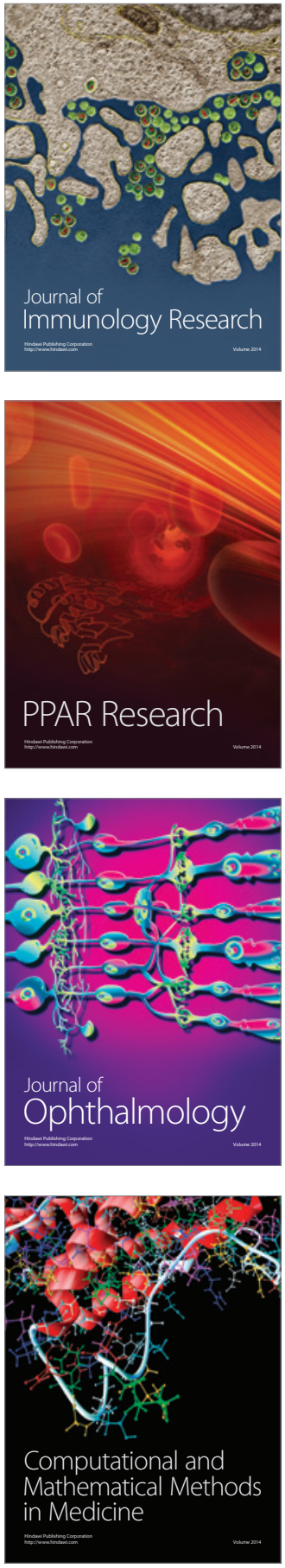

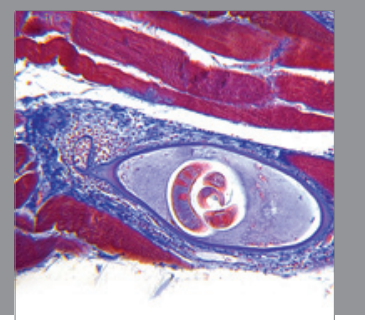

Gastroenterology

Research and Practice
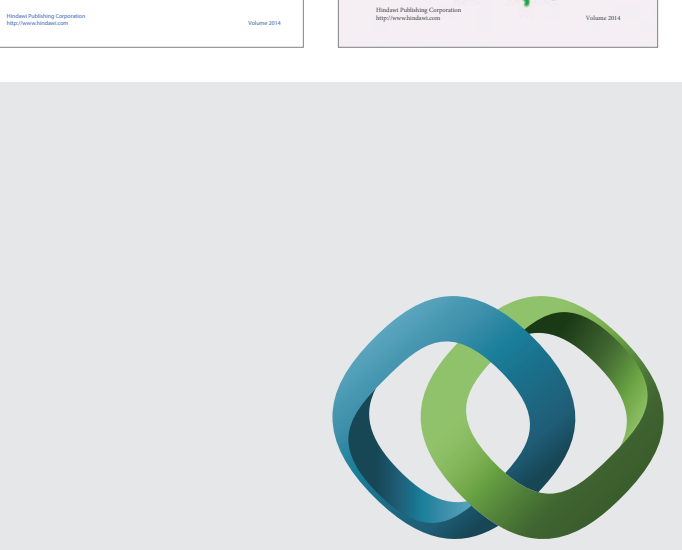

\section{Hindawi}

Submit your manuscripts at

http://www.hindawi.com
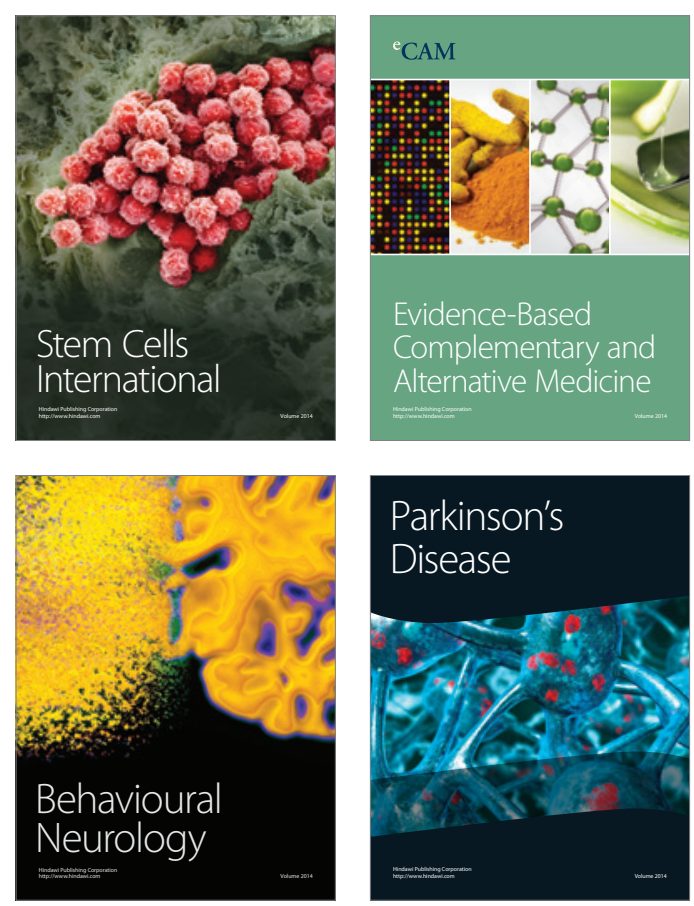

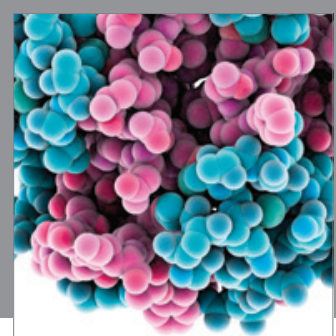

Journal of
Diabetes Research

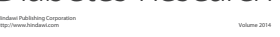

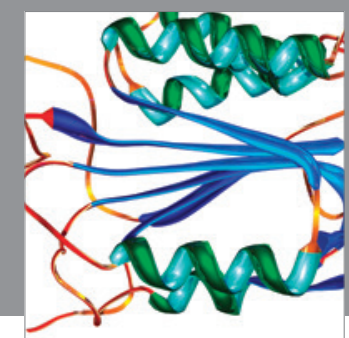

Disease Markers
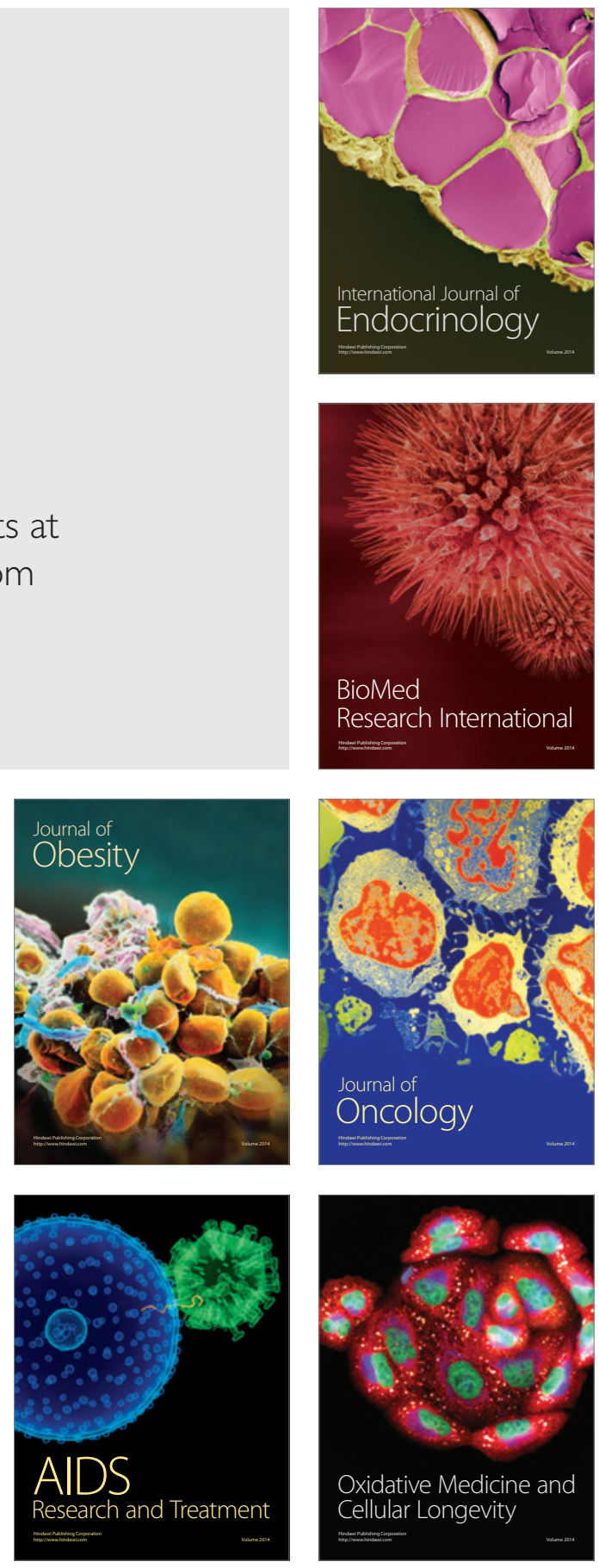